\title{
MODIFICAÇõES AGUDAS DOS NÍVEIS SÉRICOS DE CREATINA QUINASE EM ADULTOS JOVENS SUBMETIDOS AO TRABALHO DE FLEXIONAMENTO ESTÁTICO E DE FORC̣A MÁXIMA
}

\author{
ACUTE CHANGES IN CREATINE KINASE SERUM LEVELS \\ IN ADULTS SUBMITTED A STATIC STRETCHING \\ AND MAXIMAL STRENGTH TEST
}

\begin{abstract}
AUTORES
Eurico Peixoto César ${ }^{1,2,3}$

Maurício Gatáz Bara Filho ${ }^{4}$

Jorge Roberto Perrout Lima ${ }^{4}$

Felipe J. Aidar

Estélio Henrique M. Dantas ${ }^{1,2}$

${ }^{1}$ Programa de Pós-graduação Stricto Sensu em Ciência da Motricidade Humana (PROCIMH) da Universidade Castelo Branco [UCB-RJ] - Rio de Janeiro - Brasil

${ }^{2}$ Laboratório de Biociências da Motricidade Humana (LABIMH) - UCB - RJ

${ }^{3}$ Docente do Curso de Educação Física da Universidade Presidente Antônio Carlos - UNIPAC - Barbacena e Leopoldina - MG

${ }^{4}$ Docente da Universidade Federal de Juiz de Fora - UFJF - MG
\end{abstract}

MODIFICAC̄õES AGUDAS DOS NÍVEIS SÉRICOS DE CREATINA QUINASE EM ADULTOS JOVENS SUBMETIDOS AO TRABALHO DE FLEXIONAMENTO ESTÁTICO E DE FORC̣A MÁXIMA 4(3): 49-55

\section{PALAVRAS-CHAVE}

flexibilidade estática; 1-RM; CK; dano muscular.

\section{KEYWORDS}

static flexibility; 1-RM; CK; muscular damage.

\section{RESUMO}

Resumo: A força e a flexibilidade são componentes presentes em um programa de treinamento, e seus valores máximos são obtidos através de testes específicos. No entanto pouco se sabe sobre seus efeitos deletérios no aparelho músculo tendíneo. Objetivo: verificar as modificações séricas de CK 24h após uma rotina de alongamentos, flexionamento estático e teste de 1-RM. Métodos: participaram do estudo 14 indivíduos de ambos os sexos (28 \pm 6 anos), divididos em grupo controle (GC $\mathbf{N}=7$ ] e grupo experimental (GE N7), sendo o último submetido a uma rotina de alongamentos [GE-AL], de flexionamento estático (GE-FLEX] e ao teste de 1-RM (GE-1-RM). A antropometria foi aferida através de uma balança digital com estadiômetro. As coletas de sangue foram obtidas utilizando-se seringas descartáveis e depositadas em recipientes de vidro vedados e enviadas ao laboratório para deteminar os níveis de CK. Utilizou-se a técnica de De Lorme e Watkins nos exercícios Supino Horizontal e Leg Press para determinar a carga de 1-RM. Realizou-se 3 séries de 20s de insistência flexionamento e 3 séries de 6 segundos de insistência para 0 alongamento. E estatística empregada foi a Prova de Friedman com post hoc de Tukey. Resultados: Os valores de CK basal e pós 24h no GC e GE (AL; FLEX e 1-RM) foram respectivamente: $195,0 \pm 129,5$ vs. $202,1 \pm 124,2$; $213,3 \pm 133,2$ vs. $174,7 \pm 115,8$; $213,3 \pm 133,2$ vs. $226,6 \pm 126,7$ e 213,3 $\pm 133,2$ vs. $275,9 \pm 157,2$. Só foi observada diferença significativa ( $\alpha=0,02$ ] nos valores do GE-1RM. Conclusão: Conclui-se que apenas os exercícios dinâmicos de força máxima foram capazes de aumentar os niveis de CK pós exercício.
ABSTRACT

Abstract: Strength and flexibility are common components of a training program and their maximal values are obtained through specific tests. However, little information about the damage effect of these training procedures in a skeletal muscle is known. Objective: To verify a serum CK changes 24h after a sub maximal stretching routine and after the static flexibility and maximal strength tests. Methods: the sample was composed by 14 subjects (man and women, 28 \pm 6 yr.) physical education students. The volunteers were divided in a control group (CG) and experimental group [EG] that was submitted in a stretching routine (EG-ST), in a maximal flexibility static test (EG-FLEX) and in 1-RM test (EG-1-RM), with one week interval among tests. The anthropometrics characteristics were obtained by digital scale with stadiometer (Filizola, São Paulo, Brasil, 2002). The blood samples were obtained using the IFCC method with reference values 26-155 U/L. The De Lorme and Watkins technique was used to access maximal maximal strength through bench press and leg press. The maximal flexibility test consisted in three 20 seconds sets until the point of maximal discomfort. The stretching was done in normal movement amplitude during 6 secons. Results: The basal and post 24h CK values in CG and EG (ST; Flex and 1 RM] were respectively 195,0 $\pm 129,5$ vs. $202,1 \pm 124,2 ; 213,3 \pm 133,2$ vs. $174,7 \pm 115,8 ; 213,3 \pm 133,2$ vs. $226,6 \pm 126,7$ e 213,3 \pm 133,2 vs. $275,9 \pm 157,2$. It was only observed a significant difference $(\alpha=0,02)$ in the pre and post values inGE-1RM. Conclusion: only maximal strength dynamic exercise was capable to cause skeletal muscle damage. 


\section{INTRODUC̣ÃO}

Os treinamentos de força e flexibilidade são componente comumente presentes em programas de exercícios físicos ${ }^{30}$, no entanto ainda existem controvérsias sobre qual a maneira mais adequada de incluir ambos os elementos em um programa de treinamento. Tal fato ampara-se nas contraditórias pesquisas reportadas sobre os efeitos negativos do treinamento da flexibilidade sobre a força $a^{21,40}$ ou sobre o suposto efeito protetor da flexibilidade sobre a incidência de lesões ${ }^{1,31,38}$. Inúmeras pesquisas sugerem que, praticar exercícios de flexibilidade previamente a eventos que requeiram manifestações de força e potência, repercutirá em um efeito contraproducente da performance ${ }^{15,24,29,40}$ no entanto alguns autores reportaram não ser evidente nenhum efeito negativo da flexibilidade sobre a força $a^{2,14}$.

De forma semelhante, enquanto alguns pesquisadores apontam 0 efeito protetor de níveis adequados de flexibilidade na prevenção de lesões ${ }^{16,20}$, outros concluem que a realização de exercícios de flexibilidade antes ou após práticas esportivas não reduzirá a incidência de lesões $^{18,36}$.

0 treinamento contra resistência é uma modalidade de exercício que vem crescendo em popularidade nas duas últimas décadas, particularmente devido ao seu papel em melhorar o desempenho atlético através do aumento da força muscular, potência e velocidade, hipertrofia, resistência muscular localizada (RML), performance motora, equilíbrio e coordenação $0^{2,23}$.

No entanto o dano muscular induzido pelo exercício é um fenômeno comum que resulta da prática de um exercício ao qual não se está adaptado, exercício com alto volume (séries e repetições) ou com intensidade elevada ${ }^{8}$. A lesão muscular é um dos fenômenos mais comuns provenientes de práticas esportivas, com uma incidência de $10 \%$ a $55 \%$ de todas as lesões sustentadas ${ }^{4}$.

Exercícios físicos extenuantes, quando realizados até o nível de exaustão, podem promover a formação de radicais livres que estão associados ao surgimento de danos teciduais ${ }^{9}$. Inúmeros pesquisadores têm associado a presença de certos marcadores bioquímicos na urina e no sangue à danos no aparelho músculo tendíneo $0^{5,11,26,37}$. Dentre esses marcadores bioquímicos, destacase a creatina quinase (CK), enzima encontrada predominantemente nos músculos e liberada na circulação durante lesões musculares. Portanto, a atividade sérica de CK tem sido teoricamente suposta como um marcador indireto de grande valia na fisiologia do exercício e medicina desportiva para detecção de dano muscular e over training ${ }^{27,28,37}$. Inúmeros estudos têm encontrado uma alta correlação no aumento da concentração sérica de CK pós exercícios $^{6,27}$. A maioria dos dados pertinentes ao incremento dos níveis de CK provém de estudos com corredores de longa distância, porém, exercícios de alta intensidade e curta duração parecem induzir o aumento dos níveis séricos dessa enzima, principalmente se nos mesmos forem adotadas contrações excêntricas ${ }^{37}$.

Tendo isso em vista, o presente estudo teve como objetivo verificar as modificações séricas de CK, 24h após a realização de uma rotina de alongamentos sub-máximo e dos testes máximos de flexibilidade estática e de uma repetição máxima (1-RM).

\section{METODOLOGIA}

0 presente estudo adotou o caráter analítico de corte transversal, caracterizado com estudo quase experimental. A amostra foi eleita de forma intencional, por conveniência devido à facilidade de acesso aos indivíduos. As rotinas de alongamento, flexionamento estático máximo e teste de 1-RM foram realizadas no mesmo local, sob supervisão direta dos pesquisadores. As coletas de sangue foram realizadas pela mesma enfermeira, adotando-se todos os procedimentos de higiene e cuidados como o uso de luvas e seringas descartáveis e tubos de vidro esterilizados. Todos os procedimentos utilizados nesse estudo estão em acordo com determinações institucionais $\mathrm{e}$ resolução de outubro 196/96 do Conselho Nacional de Saúde, sendo submetido e aprovado pelo Comitê de Ética em Pesquisa da Universidade Castelo Branco - UCB - RJ.

\section{Amostra}

Foram selecionados para participarem do estudo 16 indivíduos de ambos os sexos [28 \pm 6 anos], matriculados no oitavo período do Curso de Educação Física da Universidade Presidente Antônio Carlos - UNIPAC, localizada na cidade de Barbacena - MG. Após esclarecimentos fornecidos em exposição oral e escrita sobre os procedimentos inerentes ao estudo, todos os indivíduos selecionados manifestaram formalmente a concordância de participar da pesquisa através da assinatura do termo de participação livre e esclarecido.

As características antropométricas de massa corporal (MC) e estatura (EST) foram efetuadas conforme os procedimentos recomendados pela International Society for Advancement of Kineanthropometry ${ }^{25} \mathrm{e}$ 
obtidas através de uma balança digital com estadiômetro (Balança Digital Filizola, São Paulo, Brasil, 2002).

A rotina de testes máximos foi realizada na sala de ginástica do Curso de Educação Física da UNIPAC, e foram utilizados colchonetes, aparelhos de musculação, anilhas e barras de ferro (Vitally ${ }^{\circledR}$ ). A rotina de alongamento (sub-máximo] foi realizada através de três séries de seis segundos de insistência em cada um dos movimentos, com a utilização de arcos de movimento em amplitude normal, sem que os indivíduos relatassem desconforto.

Os exercícios de flexionamento estático consistiram em três séries de insistências realizadas no limiar de dor. Em cada seqüência os indivíduos foram levados, inicialmente, até o limiar de flexionamento (ponto que relataram desconforto] e se sustentou este arco de movimento por cerca de seis segundos. Seqüencialmente, se forçou o segmento corporal ata quase atingir o limiar da dor e se manteve esta amplitude de movimento por cerca de 10 a 15 segundos, perfazendo um total de 15 a 20 segundos por insistência.

Para se obter os valores de força máxima foi empregada a técnica de De Lorme e Watkins (1948) e os exercícios eleitos foram o supino horizontal e o leg press $45^{\circ}$.

As coletas de sangue foram obtidas utilizando-se seringas descartáveis e as amostras foram depositadas em recipientes de vidro vedados, cedidos pelo laboratório. Para se determinar os níveis de CK, foi adotado o método IFCC, com o padrão de referência de 26 a 155 U/I e todas as análises das amostras de sangue foram realizadas no Instituto Hermes Pardini - Belo Horizonte - MG, utilizando-se o método calorimétrico.

\section{Procedimentos}

Previamente aos testes, foi efetuada uma anaminese nos voluntários da pesquisa a fim de certificar-se que nenhum deles apresentava quadro de inflamação, dor ou lesão mio-articular.

Os indivíduos foram divididos em dois grupos; grupo experimental [ $G E=9 ; 5$ homens e 4 mulheres] que realizou a série de alongamentos [GE-AL], a rotina de testes máximos de 1-RM (GE-1-RM) e rotina de flexionamento estático [GE-FLEX] em semanas distintas e o grupo controle $[G C=7 ; 4$ homens e 3 muIheres], que não realizou nenhuma intervenção física durante o período de coletas, seguindo apenas os padrões para coleta de sangue, estipulados pelos presentes investigadores.

Os indivíduos foram orientados a fazer um jejum de 12h previamente às coletas de sangue. A rotina de testes máximos e coletas de sangue foram efetuadas no período da manhã. Os exercícios de alongamento, de flexionamento estático e de 1-RM foram realizados em momentos distintos, com intervalo mínimo de uma semana entre eles. O GE efetuou 6 coletas de sangue: uma coleta basal, pré flexionamento estático e uma coleta $24 \mathrm{~h}$ após
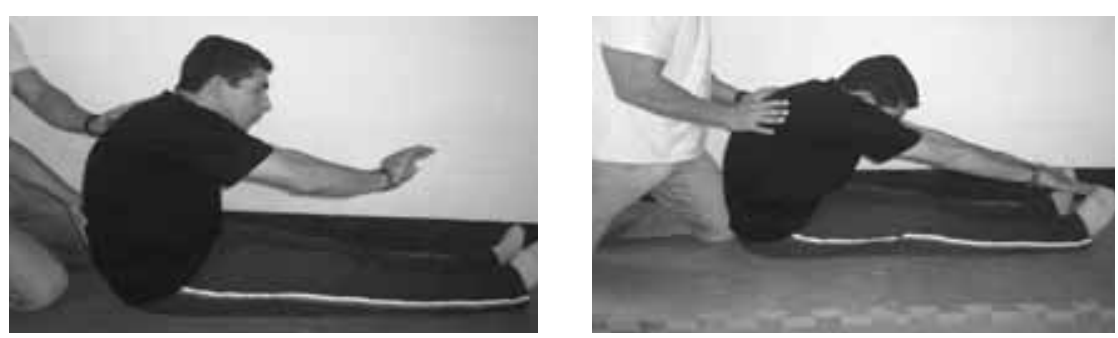

FIGURA1

FIGURA?

Flexão da coluna lombar; Posição 1. essa rotina. 0 mesmo padrão foi adotado para o teste de força máxima e para rotina de alongamentos. O GC realizou duas coletas de sangue: uma coleta basal e outra $24 \mathrm{~h}$ após coleta basal, sem nenhuma intervenção física entre elas.

Os exercícios de alongamento consistiram em: (a) abdução da articulação do ombro com cotovelo fletido; (b) flexão da perna; (c) flexão horizontal da articulação do ombro, (d) flexão do quadril com a perna flexionada e (e) flexão da coluna lombar com as pernas estendidas. Todos os exercícios foram realizados dentro dos limites normais de amplitude de movimento (ADM), em posição ortostática, com três séries de seis segundos de insistência, com cinco segundos de intervalo entre cada série.

A rotina de flexionamento procedeu da seguinte forma: os indivíduos foram todos dispostos em duplas em uma mesma sala, e sob a supervisão do avaliador, foram orientados a realizar o flexionamento estático máximo no seu companheiro, utilizando-se três séries de 15-20 segundos de insistência, contados somente após ser atingido o ponto de maior desconforto manifestado pelo testado. 
Os exercícios consistiram em: (a) extensão horizontal da articulação do ombro com o cotovelo estendido; (b) abdução da articulação do ombro com cotovelo fletido; (c) flexão da coluna lombar na posição sentado com as pernas estendidas; (d) flexão do quadril em decúbito dorsal com a perna estendida. Todos os exercícios foram realizados em amplitude máxima de movimento (AMDM).

0 teste de 1-RM seguiu o seguinte padrão: os avaliados foram orientados a realizarem um aquecimento específico no próprio aparelho onde seria feito o teste, com uma carga confortável para realização de 15 repetições. Após 5 minutos de intervalo adicionou-se carga ao aparelho e o avaliado foi instruído a realizar uma repetição máxima. À medida que o indivíduo conseguia vencer a resistência oferecida pelo aparelho, eram acrescidos de 1,8 a 4,5kg à sobrecarga, por no máximo seis tentativas, com um intervalo de dois a três minutos entre elas (ACSM, 2003; p. 380). Ao se obter a carga de 1-RM no exercício de supino horizontal, foi realizado o teste de 1-RM com o mesmo critério no exercício de Leg Press $45^{\circ}$.

Quanto à ADM dos exercícios, no supino horizontal o testado foi orientado a partir de extensão total dos cotovelos; descer a barra controladamente até que tocasse o osso externo, e retornar a posição inicial. No Leg Press $45^{\circ}$, o indivíduo foi instruído a iniciar com extensão total dos joelhos, descer até ser formado um ângulo de $90^{\circ}$ entre a perna e a coxa, e retornar ao ponto de partida. Para assegurar que a angulação de $90^{\circ}$ no movimento fosse atingida, foi fixado um goniômetro em um dos membros inferiores do testado e feita uma marcação no aparelho, indicando até onde deveria ser realizado o exercício.

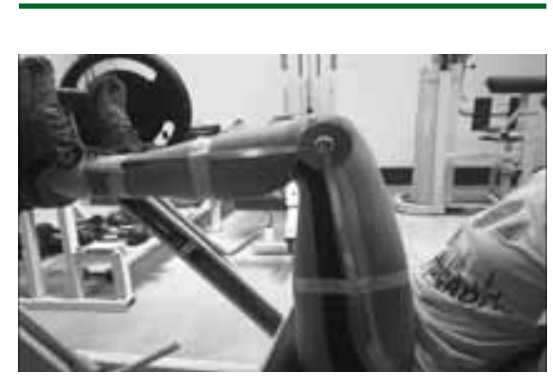

FIGURA3

Aferição da angulação estipulada no teste de 1-RM no leg press.

As amostras de sangue foram submetidas ao laboratório em um prazo máximo de duas horas, para análise de CK.

\section{Estatística}

Utilizou-se a estatística descritiva para caracterizar os indivíduos da amostra. Para comparação das médias de $\mathrm{CK}$ basal e pós $24 \mathrm{~h}$ às rotinas de flexionamento estático, teste de 1-RM e exercícios de alongamento, empregou-se o teste não paramétrico conhecido como Prova de Friedman, devido ao pequeno $\mathbf{N}$ amostral, e o post hoc de Tukkey para identificar as possiveis diferenças. Ainda empregou-se o teste de Levene para se verificar a gaussianidade da amostra.

Todas as análises foram efetuadas no pacote estatístico Statistica 6.0 (Statsoft. Inc., Tulsa, USA, 2001).

\section{RESULTADOS}

Após realizados os testes e as coletas, e ao se fazer a análise dos dados, os presentes pesquisadores entraram no consenso em excluir da amostra dois indivíduos do GE, pois os mesmos foram identificados como out liers, ficando dessa forma, um $\mathbf{N}$ válido de sete indivíduos para o GE e sete indivíduos para o GC.

Na tabela 1 estão reportados os valores de CK basal e $24 \mathrm{~h}$ referentes aos Grupos Experimental e Controle.

A figura 1 refere-se aos valores de CK basal e pós 24h no GE (1-RM, FLEX e AL], identificados pela seguinte legenda: GE-1-RM basal e 24h (preto); GE-FLEX basal e 24h (verde escuro) e GE-AL basal e $24 \mathrm{~h}$ (verde).

\section{DISCUSSÃO}

0 presente estudo buscou identificar, através do marcador bioquímico indireto creatina quinase (CK), se a prática de alongamentos (sub-máximos) e testes máximos de força (1-RM) e Flexibilidade (flexionamento estático), são lesivos ao aparelho músculo tendíneo. Pretende-se com a interpretação dos resultados obtidos, sugerir ou não a realização de tais procedimentos, amplamente difundidos tanto em âmbito profissional quanto recreacional, e desta forma propiciar infor-mações acerca dos testes e práticas mais adequadas a serem realizados.

As qualidades físicas força e flexibilidade são componentes comumente visados em um programa de treinamento ${ }^{30}$. Da mesma forma, 0 alongamento, em sua forma sub-máxima, é usualmente recomendado e adotado por técnicos, atletas, fisioterapeutas e educadores físicos como forma de aquecimento ${ }^{12}$, prevenção de lesões ${ }^{36}$ e incremento na performance ${ }^{37}$, apesar de ainda existirem controvérsias sobre esses temas $^{30,34 \text {. }}$. 
A prescrição da intensidade no treinamento contra resistência (TCR) tanto em academias quanto em centros especializados de treinamento é usualmente baseado no número de repetições máximas (RM) ou na porcentagem do valor referente a uma repetição máxima (1-RM) ${ }^{16}$.

De forma semelhante, inúmeras modalidades esportivas requerem niveis excepcionais de flexibilidade, que são conseguidos através de diversos tipos de treinamentos, dentre eles o método estático má$x^{x i m o}{ }^{10}$. No presente estudo, não foram encontrados aumentos significativos $(p>0,05)$ nos valores de CK 24h após os exercícios de alongamento (sub-máximo) e pós rotina de flexionamento estático. No entanto observou-se um aumento significativo $(\alpha=0,02)$ deste marcador 24h pós-teste de 1-RM em relação a todos os grupos em estado basal (GE 1-RM, FLEX, AL e GC). Tal fato sugere que a CK é um marcador sensivel a grandes manifestações de força, os quais são observados nos testes de 1-RM.

Inúmeros pesquisadores têm reportado o aumento dos níveis séricos de CK pós exercícios físicos ${ }^{6,37}$. No entanto, a maioria das pesquisas reportadas sobre essa condição se ampara em exercícios excêntri$\cos ^{7,26,33}$ ou exercícios de enduran$c e^{11,37}$, sendo escassas publicações sobre o comportamento dessa enzima frente a testes máximos de força dinâmica e de flexibilidade.

Um dos possíveis motivos pelo qual a CK não se apresentou aumentada pós flexionamento estático ampara-se no fato desse tipo de trabalho incidir prioritariamente sobre as articulações e não sobre o tecido muscular, como sugere Dantas ${ }^{13} \mathrm{e}$ Soares $^{32}$.

\begin{tabular}{lcc}
\hline & CK basal & CK 24h \\
\hline GE 1-RM & $213,3 \pm 133,2$ & $\star 275,9 \pm 157,2$ \\
GE FLEX & $213,3 \pm 133,2$ & $226,6 \pm 126,7$ \\
GE AL & $213,3 \pm 133,2$ & $174,7 \pm 115,8$ \\
GC & $195,0 \pm 129,5$ & $202,1 \pm 124,2$ \\
\hline
\end{tabular}

TABELA1

Dados descritivos do Grupo Experimental e Grupo Controle referentes aos valores de CK basal e $24 \mathrm{~h}$.

* valor significativo
Uma questão a ser levantada refere-se à predominância do tipo de fibra muscular e a diferença entre os gêneros. De acordo com Totsuka et al. ${ }^{37}$, o conteúdo e composição de tecido conjuntivo se diferem entre os tipos de fibras musculares, onde as fibras do tipo I apresentam uma estrutura de tecido conjuntivo mais robusta do que as fibras do tipo II, sugerindo dessa forma uma maior susceptibilidade de lesão por estiramento e tensão excessiva desta última.

Não obstante a esse fato, Stupka et al. $^{35}$ sugerem haver respostas diferentes entre os gêneros, no que diz respeito a dano muscular gerado por exercícios, sendo que os indivíduos do sexo feminino apresentam menor inflamação muscular em relação aos homens.

No presente estudo, os indivíduos não foram divididos em relação à predominância de fibras musculares ou em relação ao sexo. Porém observou-se uma tendência dos indivíduos do sexo masculino em serem mais responsivos ao teste de 1-RM, apresentando maiores variações de CK pós-exercício, o que vai ao encontro do estudo de Stupka et al. ${ }^{35}$.

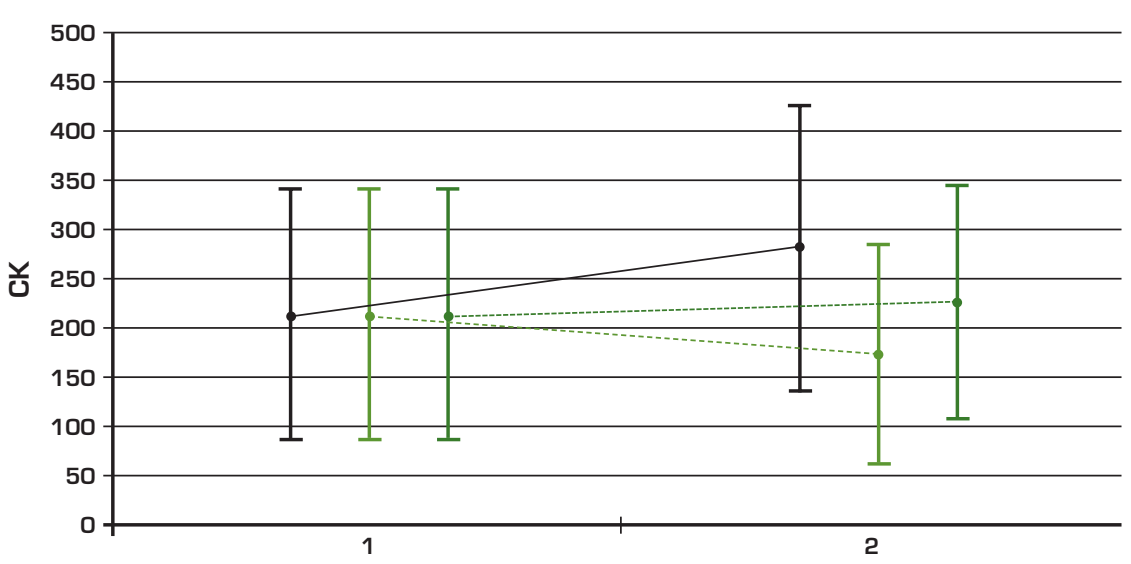

FIGURA4

Valores de CK basal e 24h referentes ao GE. 
Outro fator que merece atenção é o fato de que a resposta de CK aos exercícios dependa das características físicas e de composição corporal, além do histórico de treinamento de cada indivíduo. Tal fato é apreciado em um estudo de Totsuka et al. ${ }^{37}$, onde os indivíduos mais responsivos aos exercícios físicos, ou seja, aqueles que apresentaram maiores valores de CK pós-exercício, foram aqueles dotados de menor massa muscular e força. Esse dado sugere que indivíduos mais fracos ou de menor compleição física tendem a apresentar danos acentuados no tecido muscular após prática de exercícios extenuantes ou máximos.

No presente estudo os indivíduos não foram separados de acordo com suas características antropométricas e composição corporal, nem em relação ao status de treinamento, o que provavelmente pode ter influenciado nos resultados encontrados. Além disso, o $\mathbf{N}$ amostral relativamente pequeno dificulta qualquer inferência mais acurada sobre os resultados encontrados no presente estudo.

No entanto, o fato dos valores de CK apresentarem um aumento significativo $24 \mathrm{~h}$ após o teste de 1-RM no GE 1-RM, em relação aos outros grupos em estado basal, vêm contribuir de forma categórica no entendimento do papel do teste de força dinâmica máxima na intercorrência de danos ao tecido muscular.

\section{CONCLUSÃO}

Com base nos dados obtidos no presente estudo, pode-se concluir que os exercícios sub-máximos de alongamento e os exercícios de flexionamento estático não apresentaram diferença significativa
( $p>0,05$ ) nos valores de CK basal e pós $24 h$, o que sugere uma ineficácia desses procedimentos em causar danos importantes no tecido muscular. No entanto, observou-se um aumento significativo $(\alpha=0,02)$ nos níveis de CK pós teste de 1-RM, sugerindo desta forma que, exercícios dinâmicos, realizados com intensidade próxima aos 100\% da força máxima, podem gerar danos no aparelho músculo esquelético.

A partir do conhecimento de que, no presente estudo não houve uma distinção da amostra em relação ao sexo, nível de treinamento ou variáveis morfológicas, o que pode ter influenciado os resultados encontrados, sugere-se para novas investigações, que sejam adotados esses procedimentos, além de outras formas de flexibilidade máxima, como a dinâmica, por exemplo.

\section{CORRESPONDÊNCIA}

Eurico P. César

R. Dr. Alberto Vieira Lima, $n \div 82$

Bairu - CEP 36050-070 - [32]

3212 2304; Juiz de Fora - MG

\section{REFERÊNCIAS}

1. Alter MJ (1997). Science of Flexibility. Champaign, IL: $2^{\underline{a}}$ ed. Human Kinetics.

2. Lopes AC; Barreto G; Vale RGS; Novaes JS. Comparação nos níveis de flexibilidade entre praticantes de musculação e ginástica localizada. Motricidade, 2(3)315-321.

3. Behm DG; Bradbury EE; Haynes AT et al. [2006). Flexibility is not related to stretch-induced deficits in force or power. J Sports Sci Med 5:33-42.
4. Beiner JM; JokI P [2001). Muscle contusion injuries: current treatment options. J Am Acad Orthop Surg 9:227-237.

5. Bikle DD (1997). Biochemical markers in the assessment of bone disease. Am J Méd 103:427-436.

6. Brancaccio P; Limongelli FM; Maffulli N (2006). Monitoring of serum enzymes in sport. $\mathrm{Br} J$ Sports Med 40:96-97.

7. Byrne C; Eston RG; Edwards RHT [2001). Characteristics of isometric and dynamic strength loss following eccentric exercise-induced muscle damage. Scand J Med Sci Sports 11:134-140.

8. Byrne C; Twist C; Eston R (2004). Neuromuscular function after exercise-induced muscle damage theoretical and applied implications. Sports Med 34(1):49-69.

9. Cabrera MCG; Pallardó FV; Sastre J; Viña J; Moral LGdel (2003). Allopurinol and markers of muscle damage among participants in the tour de France. JAMA 289(19):2503-2504.

10. Chan SP; Hong Y; Robinson PD (2001). Flexibility and passive resistance of the hamstrings of young adults using two different static stretching protocols. Scand J Med Sci Sports 11:81-86.

11. Clarkson PM; Sayers SP (1999). Gender differences in exerciseinduced muscle damage. In: Gender Differences in Metabolism: Practical and Nutritional Implications, edited by Tarnopolsky MA. Boca Raton, FL: CRC, 283-299.

12. Cornwell A; Nelson AG; Heise GD; Sideway B (2001). The acute effects of passive muscle stretching on vertical jump performance. J Hum Mov Stud 40:3 07-324.

13. Dantas EHM (2005). Flexibilidade, alongamento e flexionamento. Rio de Janeiro: $3^{\text {a }}$ ed. Shape. 
14. Egan AD; Cramer JT; Massey LL et al. (2006). Acute effect of static stretching on peak-torque and mean power output in national collegiate athletic association division I women's basketball players. J Strength Cond Res 20(4):778-82.

15. Evetovich TK; Nauman NJ; Conley DS; Todd JB (2003). Effect of static stretching of the biceps brachii on torque, electromyography, and mechanomyography during concentric isokinetic muscle actions. J Strength Cond Res 17: :484-488.

16. Fleck SJ; Kraemer WJ (2004). Designing Resistance Training Programs. Champaign, IL: $3^{\text {rd }}$ ed. Human Kinetics Publishers.

17. Garrett WE Jr (1996). Muscle strain injuries. Am J Sports Med; 24:S2-58.

18. Gleim GW; McHugh MP (2002). Flexibility and its effects on sports injury and performance. Sports Med 24:289-299.

19. Herbert RD; Gabriel M (2002). Effects of stretching before and after exercising on muscle soreness and risk of injury: a systematic review. Br Med J 325:468-470.

20. Jonhagen S; Nemeth G; Eriksson E (1994). Hamstrings injuries in sprinters: the role of concentric and eccentric hamstring muscle strength and flexibility. Am J Sports Med. 22:262-2666.

21. Kokkonen J; Nelson AG; Cornwell A (1998). Acute muscle stretching inhibits maximal strength performance. Res Q Exerc Sport 69 (4): 411-415.

22. Koskinen SO; Ho"Yhtya" M; Turpeenniemi-Hujanen T; Martikkala V; Ma"Kinen TT; Oksa J. et al. (2001). Serum concentrations of collagen degrading enzymes and their inhibitors after downhill running. Scandinavian Journal of Medicine and Science in Sports 11:9-15.
23. Kraemer WJ; Ratamess NA (2004). Fundamentals of resistance training: progression and exercise prescription. Med Sci Sports Exerc 36(4):674-688.

24. Marek SM; Cramer JT; Fincher AL; Massey LL; Dangelmaier SM; Purkayastha S; Fitz KA; Culberrtson JY (2005). Acute effects of static and proprioceptive neuromuscular facilitation stretching on muscle strength and power output. J Athl Train 40:94-103.

25. Norton K; Olds T (1996). Anthropometrica. Sidney, Australia: University of New South Wales Press. 26. Paschalis V; Koutedakis Y; Jamurtas AZ; Mougios V; Baltzopoulos $V$ (2005). Equal volumes of high and low intensity of eccentric exercise in relation to muscle damage and performance. J Strength Cond Res 19(1):184-188.

27. Purge $P$; Jurimae J; Jurimae $T$ (2006). Hormonal and psychologicoal adaptationin elite male rowers during prolonged training. J Sports Sci 24 (10):1075-1082.

28. Raastad T; Glomsheller T; Bjoro T; Hallén J (2003). Recovery of skeletal muscle contractility and hormonal responses to strength exercise after two weeks of high-volume strength training. Scan J Med Sci Sports 13:159-168.

29. Rubini EC; Pereira MIR; Gomes PSC (2004). Acute effect os static and pnf stretching on hip adductor isometric strebgth. Med Sci Sports Exerc 36(8):1389-96.

30. Rubini EC; Costa ALL; Gomes PSC (2007). The effects of stretching on strength performance. Sports Med 37 (3):213-224.

31. Smith CA (1994). The warm-up procedure: To stretch or not to stretch: A brief review. J Orthop Sports Phys Ther 19:12-17.

32. Soares WD, Santos RS, Almeida FN, Neto JTM, Novaes JS.
[2005). Determinação dos níveis de flexibilidade em atletas de Karatê e jiujitsu. Motricidade 1(4):246-252. 33. Sorichter S; Mair J; Koller A; Müller E; Kremser C; Judmaier W et al. (2001). Creatine kinase, myosin heavy chains and magnetic resonance imaging after eccentric exercise. Journal of Sports Sciences 19:687- 691.

34. Stone M; Ramsey MW; Kinser AM; O'Bryant HS; Ayers C; Sands WA (2006). Stretching: acute and chronic? the potential consequences. National Strength and Conditioning Association 28(6):66-74. 35. Stupka N; Lowther S; Chorneyko $\mathrm{K}$; Bourgeois JM; Hogben C; Tarnopolsky MA (2000). Gender differences in muscle inflammation after eccentric exercise. J Appl Physiol 89:2325-2332.

36. Thacker SB; Gilchrist J; Stroup DF; Kimsey Jr. CD (2004). The impact of stretching on sports injury risk: a systematic review of the literature. Med Sci Sports Exerc 36(3):371-378.

37. Totsuka M; Nakaji S; Suzuki K; Sugawara K; Sato K (2002). Break point of serum creatine kinase release after endurance exercise. J Appl Physiol 93:1280-1286.

38. Weerapong P; Hume PA; Kolt GS (2004). Stretching: mechanisms and benefits for sport performance and injury prevention. Physical Therapy Reviews 9:189-206.

39. Willems MET; Stauber WT (2001). Force deficits after repeated stretches of activated skeletal muscles in female and male rats. Acta Physiol Scand 172:63-67.

40. Yamaguchi T; Ishii K; Yamanak $M$ et al. (2006). Acute effect of static and flexibility stretching on power output during concentric dynamic constant external resistance leg extension. J Strength Cond Res 20 (4):804-810. 